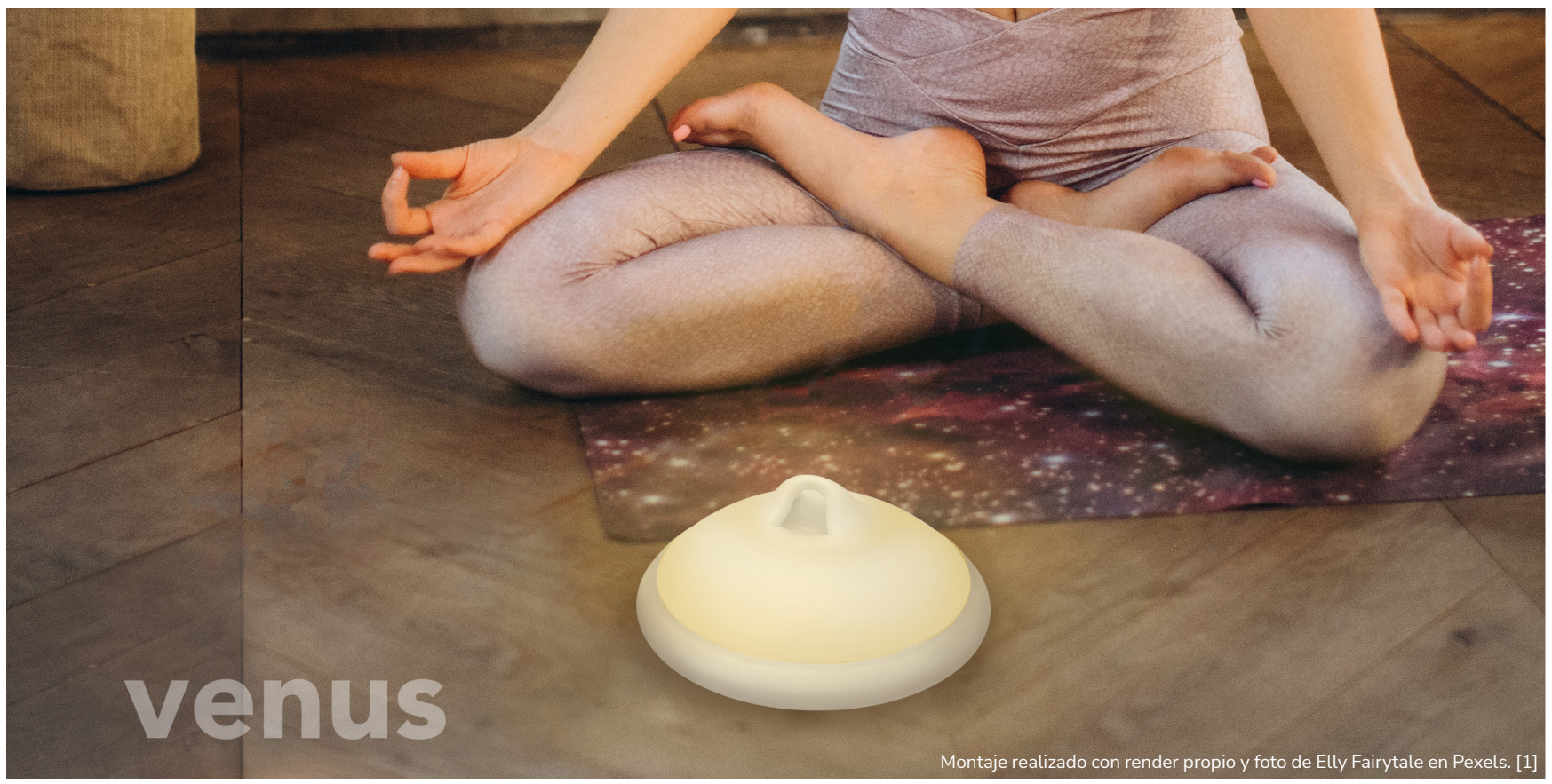

\title{
Conceptualización de luminaria solar para aliviar la dismenorrea en personas menstruantes a través del calor y la meditación
}

Solar luminaire conceptualization for the dysmenorrhea relief in menstruating people through the heat and meditation

Andrea M. Rojas-Jiménez ${ }^{1}$, Katherina Jurburg-Chaves ${ }^{2}$, Michelle Lacouture-Solís ${ }^{3}$

A. Rojas-Jiménez, K. Jurburg-Chaves, M. Lacouture-Solis "Conceptualizaci\{on de luminaria solar nómada para aliviar la dismenorrea en personas menstruantes a través del calor y la meditación", IDI+, vol. 2, no 2, Ene., pp. 4-19, 2022.

doi) https: //doi.org/10.18845/ridip.v4i2.6070 Fecha de recepción: 30 de junio de 2021

Fecha de aprobación: 2 de diciembre de 2021

1. Andrea M. Rojas-Jiménez

Estudiante de Ingeniería en Diseño Industrial Instituto Tecnológico de Costa Rica Cartago, Costa Rica androji.13@gmail.com (D) 0000-0001-5613-6984
2. Katherina Jurburg-Chaves

Estudiante de Ingeniería en Diseño Industrial Instituto Tecnológico de Costa Rica Cartago, Costa Rica yjurburg@gmail.com (D) 0000-0003-3213-935X
3. Michelle Lacouture-Solís Estudiante de Ingeniería en Diseño Industrial Instituto Tecnológico de Costa Rica Cartago, Costa Rica milaso18@gmail.com

(D) 0000-0001-5946-5839 


\section{Resumen}

La dismenorrea es un dolor uterino que sufren algunas personas menstruantes durante la fase de sangrado del ciclo menstrual. Con el propósito de disminuir dicho dolor, se desarrolló una luminaria solar, tomando como base la metodología de Design Thinking con variaciones implementadas debido a la virtualidad y las limitaciones que implicó la pandemia. Este proceso se llevó a cabo en cinco etapas: exploración de la problemática, conceptualización de la idea, desarrollo de propuestas, detallado técnico y simulación del diseño.

En la exploración e investigación, se determinó que el uso de calor en la zona abdominal aumenta el flujo sanguíneo y mejora la oxigenación de los tejidos. Asimismo, algunas posturas neutras y asanas de yoga ayudan a disminuir la dismenorrea.

A partir de estos métodos para combatir el dolor, se llevó a cabo la conceptualización de Venus, una luminaria solar nómada que integró sanación física a través del calor y sanación emocional mediante la luz. Se buscó generar calma, sanación y crear lazos emocionales entre las personas usuarias, sus ciclos menstruales y la luminaria. Esto a través de su luz tenue y cálida, que ayuda a la relajación, y una compresa caliente para ser utilizada durante la ejecución de posturas de yoga.

Este producto viene a satisfacer una necesidad de seguridad, aliviar el dolor físico de la persona menstruante con dismenorrea a través de un método no intrusivo e integral. Además, no existe una luminaria con este propósito en el mercado, de aquí la diferenciación e importancia del producto planteado.

\section{Palabras clave}

Iluminación; yoga; emocionalidad; ambientación; dismenorrea.

\section{Abstract}

Dysmenorrhea is uterine pain experienced by some menstruating people during the bleeding phase of the menstrual cycle. In order to reduce this pain, a solar luminaire was developed, based on the Design Thinking methodology with variations implemented due to the virtuality and limitations implied by the pandemic.

This process was carried out in five phases: exploration of the problem, conceptualization of the idea, development of proposals, technical details and design simulation.

In the exploration and investigation it was determined that the use of heat in the abdominal area increases blood flow and improves oxygenation of the tissues. Also, some neutral postures and yoga asanas help reducing dysmenorrhea.

From these methods to combat pain, the conceptualization of Venus was carried out, a 
nomadic solar luminary that integrated physical healing through heat and emotional healing through light. It was sought to generate calm, healing and create emotional ties between users, their menstrual cycles and the light. All this through its soft and warm light, which helps with relaxation, and a hot compress to be used during the execution of yoga postures.

This product meets a need for safety, to relif the physical pain of the menstruating person with dysmenorrhea through a non-intrusive and comprehensive method. In addition, there is no a luminnaire for this purpose on the market, hence the differentiation and importance of the raised product.

\section{Keywords}

Illumination; yoga; emotionality; ambience; dysmenorrhea.

\section{Introducción}

A partir de la menarca, primera menstruación de los cuerpos menstruantes, el útero se prepara mes a mes en caso de que un óvulo sea fecundado. Esta preparación involucra el crecimiento del endometrio. Cuando no se produce la fecundación, este se desprende y es expulsado fuera del cuerpo a través de la vagina, mientras se dan contracciones en el útero.

De acuerdo con los resultados obtenidos en el estudio de Zanin et al., en Argentina, 2010, la edad promedio de la menarca es de 12,74 años. Al mismo tiempo, se determina que los ciclos menstruales se desarrollan en el transcurso de la vida de las personas con cuerpos menstruantes durante un período de aproximadamente 40 años [2].

Dentro de este periodo, las contracciones del útero, debido al desprendimiento del endometrio, pueden causar molestias y dolores agudos, principalmente en las zonas del abdomen y espalda baja. A esto se le conoce como dismenorrea.

En abril de 2010, se realizó una encuesta analítica transversal en la Universidad de Módena y Reggio Emilia. Esta fue aprobada por la Junta de revisión interna del Departamento de Obstetricia, Ginecología y Pediatría, Azienda Ospedaliero Universitaria Policlínico de Módena. En este estudio realizado a 408 mujeres jóvenes (22,90 \pm 3,03 años), el 84,1 \% reportó dolor menstrual, el $43,1 \%$ indica que el dolor ocurre durante cada periodo y el $41 \%$ reporta que el dolor ocurre durante algunos períodos [3]. Asimismo, esta problemática afecta al $72 \%$ de las mujeres, según un estudio realizado en Suiza [4].

Estos porcentajes muestran que la dismenorrea es un problema real que sufren muchas personas menstruantes, y es necesario solventarlo, debido a que causa molestias que impiden el desarrollo de una vida normal durante el periodo sangrante del ciclo menstrual. Por lo cual, para el desarrollo del proyecto, se define como problemática las molestias ocasionadas por la 
dismenorrea en personas menstruantes durante la fase de sangrado del ciclo menstrual. Una de las soluciones tradicionales para aliviar el malestar que causa la dismenorrea es la aplicación de calor localizado en las zonas de dolor. Algunos de los efectos fisiológicos del calor son los siguientes: efecto vasodilatador, aumento del metabolismo tisular, efecto analgésico, antiespasmódico, antiinflamatorio, disminuye rigidez articular, aumenta extensibilidad del tejido conectivo y aumenta el flujo sanguíneo [5].

Debido a los beneficios mencionados anteriormente, en el mercado se comercializan productos que utilizan el calor para aliviar los dolores de la dismenorrea, por ejemplo, existen bolsas de caucho que funcionan como compresas en las que se introduce agua caliente y luego se colocan sobre la zona del abdomen o espalda baja. Asimismo, existen compresas de tela con semillas que se calientan en el microondas para este mismo fin.

En el estudio realizado a 92 mujeres en la Universidad Islámica de Azad [5], se demostró cómo el yoga es una alternativa no farmacológica efectiva para adolescentes con dismenorrea primaria. Existen posturas neutras y asanas de yoga que causan una reducción comprobada de la dismenorrea como en [6], donde hubo una diferencia significativa en la intensidad y la duración del dolor en el grupo experimental, pues el yoga redujo la gravedad y la duración de la dismenorrea primaria. El ejercicio aumenta el flujo de sangre a nivel pélvico, además, estimula la liberación de endorfinas que actúan como analgésicos no específicos [7].

Se implementan estas alternativas no farmacológicas (calor y posiciones de yoga) en la conceptualización y modo de uso planteado para una luminaria, dirigida a personas con cuerpos menstruantes, para inhibir la dismenorrea y ser usada en entornos domésticos o interiores. Los resultados de esta investigación, además de servir como base para el diseño de la luminaria en cuestión, tendrán un impacto positivo en la salud de las personas que lleguen a utilizar el producto, pues se aliviará su dolor físico.

\section{Metodología}

El desarrollo del proyecto se dio durante la pandemia por COVID-19, de modo que los alcances del diseño se tuvieron que adaptar a las condiciones y recursos disponibles, lo que dio como resultado una simulación 3D de la luminaria. La premisa del proyecto consistió en identificar un problema que pudiese ser solventado al conceptualizar una luminaria nómada (inalámbrica y de fácil transporte); esta debía ser impulsada por una fuente de energía renovable, además de considerar la experiencia estética, de significado y emocional del producto. La experiencia del producto se define como todo el conjunto de afectos que provoca la interacción entre una persona usuaria y un producto, incluido el grado en que todos los sentidos se satisfacen (experiencia estética), los significados que se atribuyen al producto (experiencia de significado) y los sentimientos y emociones que se obtienen (experiencia emocional) [8]. 
La metodología utilizada para el desarrollo de Venus sigue una base similar a la propuesta por el método del Design Thinking, con variaciones implementadas debido a las limitaciones por la pandemia, ya que no se desarrolló un prototipo físico y, por lo tanto, no se realizaron pruebas con personas usuarias. El proceso consistió en cinco etapas: exploración de la problemática, conceptualización de la idea, desarrollo de propuestas, detallado técnico y simulación del diseño.

\section{Exploración de la problemática}

El desarrollo de Venus inició con la exploración de distintas problemáticas que podían ser solucionadas o atenuadas mediante el uso de la iluminación para generar microambientes emocionales.

Después de una lluvia de ideas sobre dificultades presentes en la vida de distintas posibles personas usuarias, que se consideraron pertinentes para su solución a través de las propiedades de la luz, se realizó una evaluación de pros y contras de cada problema, para así seleccionar uno de gran importancia que, además, generó empatía automáticamente entre las diseñadoras.

Después, se comenzó una investigación centrada en las formas no farmacológicas de tratar la dismenorrea en cuerpos menstruantes $y$, a partir de esta, se consideraron distintos métodos para atenuar las molestias generadas por el ciclo menstrual que podrían ser utilizados en el producto posteriormente.

De la mano de estas formas no farmacológicas de tratar el dolor, se investigó acerca de posiciones de yoga que contribuyen a la reducción del dolor por dismenorrea. Para considerar la implementación de los asanas de yoga con el uso de la luminaria, se realiza una evaluación ergonómica para varias posturas, utilizando el método REBA para ambos lados del cuerpo. Este es un método de análisis postural especialmente sensible con las tareas que conllevan cambios inesperados de postura, como consecuencia normalmente de la manipulación de cargas inestables o impredecibles [9]. Se escoge este método debido a su flexibilidad para la aplicación por medio de imágenes. Lo cual es un recurso al que se debió acudir por las condiciones de confinamiento y las limitantes para realizar pruebas con posibles personas usuarias y obtener observaciones en tiempo real.

Para llevar a cabo este análisis, primero se consideró el tiempo, la frecuencia y la fuerza o carga involucrada con cada posición. Luego, con la ayuda de imágenes, se divide el cuerpo en dos grupos de acuerdo con segmentos. El Grupo A estaría conformado por el tronco, cuello y piernas. Mientras que el Grupo B se conforma del brazo, antebrazo y muñeca. Luego, dentro de cada grupo, se asignan puntuaciones que corresponden al nivel de riesgo de cada posición corporal; estas pueden cambiar si la tarea implica alguna fuerza. Posteriormente, con las puntuaciones del Grupo A y Grupo B, se obtiene una puntuación C. Esta se modifica de acuerdo con la actividad muscular y se obtiene la puntuación final. El resultado obtenido es referencia 
para el nivel de acción que se debe tomar. A pesar de ser la mayoría posiciones seguras para las personas usuarias, se escogen las posturas de padmasana para la meditación y para el caso de la postura en interacción con la luminaria, la variación del supta baddha konasana y el savasana. Estas, además de ser más cómodas para quien las realice, también son posiciones más simples y amigables para principiantes.

\section{Conceptualización de la idea}

A partir de la investigación bibliográfica donde se demuestra su eficiencia, se definieron dos métodos no farmacológicos (aplicación de calor y posiciones de yoga) para implementarlos con el uso de la luminaria. Ambos son utilizados por personas menstruantes alrededor del mundo para atenuar los dolores de la dismenorrea. Basado en esto y la problemática identificada, se procede a definir los objetivos del proyecto. Como objetivo general, se espera desarrollar una luminaria que combine métodos de meditación con estímulos físicos para transmitir bienestar físico y mental a las personas menstruantes durante la fase de sangrado de su ciclo menstrual. Este se lograría a través de tres objetivos específicos:

- Diseñar una luminaria nómada para personas menstruantes.

- Generar un producto que incorpore técnicas de meditación en su uso para generar una sensación de bienestar en la persona usuaria.

- Utilizar la luz acompañada de estímulos físicos para aliviar la dismenorrea.

El producto estaría dirigido a personas menstruantes de todas las tallas y colores, que sufren de dismenorrea, mejor conocida como dolores menstruales. Las cuales desean aliviar su dolor en el abdomen, espalda o busto. Además, buscan relajarse y sentirse serenas mientras están en sus hogares con el periodo menstrual.

Junto a todos estos detalles, también se plantearon la emocionalidad, proyección de la luz, aspectos perceptuales y funcionales del producto. Además, se delimitaron los requerimientos para el desarrollo de la luminaria. Estos elementos se utilizaron como norte para el desarrollo del proyecto, y con ellos se definió la morfología, partes y materiales del producto por diseñar.

\section{Desarrollo de propuestas}

Inicialmente, se desarrollaron seis distintas propuestas de diseño, a través de bocetos rápidos, donde se tomaron en cuenta los aspectos mencionados antes. Después, se realizó una discusión sobre los principales pros y contras tomando en cuenta los criterios establecidos en los requerimientos de diseño. En dicha discusión, también se consideraron aspectos como la distribución interna de componentes, sistema de sujeción, estética y ensamblaje. Lo que permitió rescatar características positivas de cada diseño y plasmarlas en una nueva propuesta, con la cual, después de anotaciones y cambios, se llegó a un octavo y último diseño. Este se convirtió en la propuesta base de la luminaria, la cual fue modificada posteriormente durante 
el desarrollo del proyecto para satisfacer los requerimientos de una manera más efectiva. A partir de la propuesta seleccionada, se realizó un modelado volumétrico de cinco diseños con la misma configuración y componentes, pero con variaciones morfológicas para una evaluación perceptual a través de Google Forms con posibles personas usuarias.

\section{Detallado técnico}

Por medio de la herramienta SolidWorks, se realizó el modelado 3D de la luminaria. Con este se generaron los planos técnicos, donde se muestran detalles importantes acerca de la morfología de la luminaria. También, se realizaron esquemas a modo de infografías para describir su configuración, partes y materiales.

\section{Simulación del diseño}

Para finalizar el proceso de diseño, se realizaron renders de alta calidad, videos y fotomontajes por medio de la herramienta KeyShot. Con estos se ilustra la configuración, ensamblaje, funcionalidad y emocionalidad del producto. Además, un detalle importante de Venus fue el efecto luz, ya que esta es la responsable de lograr el microambiente, alcanzar la relajación y sanación de la persona que la está utilizando. Por medio de la simulación de diseño, fue posible verificar que se cumpliera con los requisitos técnicos y perceptuales.

\section{Resultados}

Tras la investigación, se decide tratar el problema definido anteriormente y utilizar dos métodos atenuantes del dolor: el tratamiento con calor y los asanas de yoga. A continuación, se describe el aporte de cada una de estas técnicas en el tratamiento de los dolores menstruales.

\section{Aplicación de calor focalizado}

Se ha demostrado que usar calor a nivel abdominal $\left(39 \mathrm{C}^{\circ}\right)$ durante 12 horas se considera tan efectivo como el ibuprofeno ( $400 \mathrm{mg}, 3$ veces al día) [5]. Por lo tanto, se propone un producto que integre el uso del calor para la disminución del dolor generado por el ciclo menstrual.

El calor actúa inhibiendo las acciones de los nervios simpáticos, de esta manera, se aumenta el flujo sanguíneo y así las sustancias productoras del dolor como las prostaglandinas y los leucotrienos son removidas con mayor rapidez. Además, este aumento del flujo sanguíneo mejora la oxigenación de los músculos y evita la generación de isquemia [10]. Debido a esta función como excelente relajante muscular, los efectos en las personas usuarias son positivos, pues generan calma y confort, de manera que los malestares relacionados al ciclo menstrual disminuyen significativamente.

Estos beneficios se incorporaron al producto diseñado a través de la implementación de una compresa de tela removible, rellena de linaza y apta para microondas, que permite a la persona 
usuaria realizar una terapia del calor de forma práctica y segura. Al ser porosa y estar en contacto con el vientre de la persona usuaria, esta compresa está diseñada para ser lavada y reemplazada en caso de que se necesite.

\section{Asanas de Yoga}

Se evaluaron las posturas que representaban una postura neutra, aspecto necesario para realizar la terapia de calor simultáneamente; pavanamuktasana, balasana, supta baddha konasana y savasana. Se realiza la evaluación ergonómica de carga postural de estas variantes (figura 1). Por otro lado, durante la meditación, se recomienda adoptar las posturas de padmasana o siddhasana, pues la persona usuaria puede colocar la luminaria en una superficie mientras medita acompañada de la luz en esta posición.

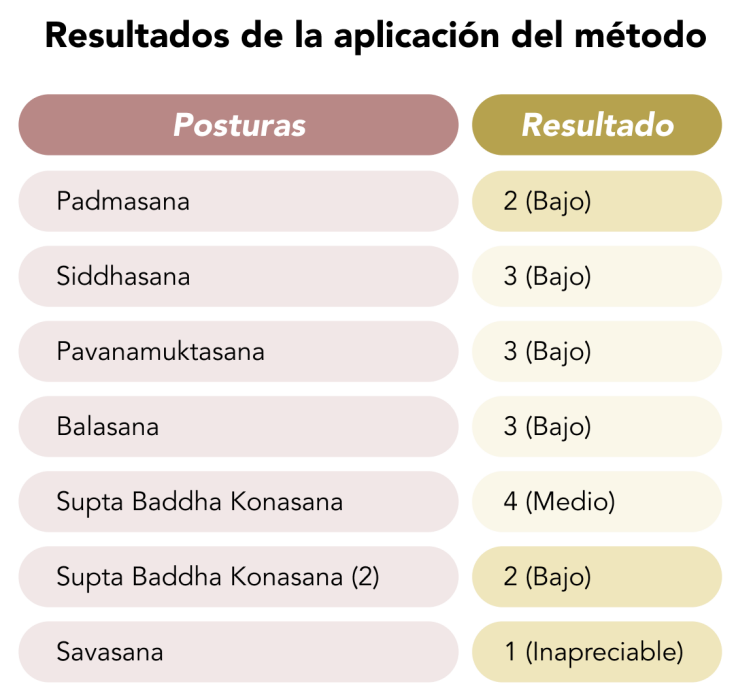

Fig. 1. Evaluación ergonómica de las Asanas de yoga.

Al diseñarse una luminaria nómada, se consideró que esta tuviera un peso liviano, pues, como lo dice su nombre, estará en constante cambio de posición. De acuerdo con la guía de levantamiento de carga GINSHT, guía técnica para la evaluación y prevención de los riesgos relativos a la manipulación manual de cargas [11], se considera que una carga por debajo de 3 kg no representa un riesgo ergonómico.

Para establecer el peso máximo de la luminaria, se toma como referencia el peso de compresas y de luminarias nómadas existentes. De la muestra analizada, el promedio de peso fue de 1,13 $\mathrm{kg}$. El valor mínimo fue de 0,32 kg, mientras el máximo fue de 2,2 kg, por ende, se concluye que el producto debe tener un peso menor al promedio.

Para el tamaño de la luminaria, se considera el rango entre la medida del ancho de los órganos reproductores internos de una persona menstruante adulta sin partos (13 cm con holgura) [11] y la medida de ancho de cadera de una mujer de 25 a 34 años percentil 50 (35,6 cm) [12]. 
Para el diseño de la luminaria, se plantea el concepto sanación cíclica, el cual hace referencia a la curación periódica del cuerpo menstruante por medio de terapias alternativas. Este concepto incluye el entorno doméstico donde se utilice Venus, un ambiente que propicie el descanso y relajación (sanación); asimismo, en la emocionalidad que genera en la persona usuaria mientras medita, gracias a la proyección tenue de la luz y la calidez de esta. Además, este concepto sirvió durante el desarrollo del proyecto como una guía para la definición formal del producto, una forma circular y continua (cíclica).

En este concepto, se abordaron los aspectos más importantes a tomar en cuenta durante el diseño del producto. En este caso, fue de vital importancia definir el entorno de uso, debido a que el producto estaba destinado a crear un microambiente de sanación y a acompañar a la persona usuaria mientras medita o descansa durante las fases del ciclo menstrual donde sienta malestar, por esta razón, tendrá un uso doméstico y su morfología debe facilitar su transporte dentro del hogar, lo cual es parte de su característica nómada.

Se define una proyección de la luz cálida y tenue de 3000 K, pues esta temperatura se relaciona con relajación y con la luz del fuego de la candela que se utiliza para la meditación; una luz de temperatura neutra o fría podría encandilar, incomodar a la persona usuaria y, por ende, agravar su malestar. Partiendo de esta característica, se pretende que su uso propicie el bienestar físico y mental, a través del calor y la meditación respectivamente. Además, al ser parte del proceso de sanación, la persona usuaria podrá desarrollar un vínculo emocional con la luminaria.

Para garantizar una experiencia estética placentera, se considera el uso de materiales livianos, que se perciban suaves y cálidos al tacto. Se desea comunicar el concepto de sanación y confort con el uso de materiales opacos sin poros, colores claros que se puedan asociar con el bienestar mental y suavidad, texturas lisas y blandas (con el uso de materiales con alto grado de deformación elástica) para que la luminaria se adapte al cuerpo de la persona usuaria. También se emplea una topología curva con superficies regladas sin grados de continuidad marcados (transiciones suaves) para transmitir gentileza. Además, el uso de materiales de poco peso garantizará el uso seguro de la luminaria sobre el vientre en las posturas de savasana y supta baddha konasana.

Asimismo, se consideran los aspectos más relevantes de la funcionalidad, los cuales son la aplicación del calor a una temperatura ideal de $39^{\circ} \mathrm{C}$, se ha demostrado que su uso a nivel abdominal se considera tan efectivo como el ibuprofeno [5], por ende, los materiales utilizados en la fabricación de la luminaria deben ser resistentes al calor.

Definidos estos aspectos durante el uso de la luminaria, se realizaron los ocho bocetos que se muestran en la figura 2. 

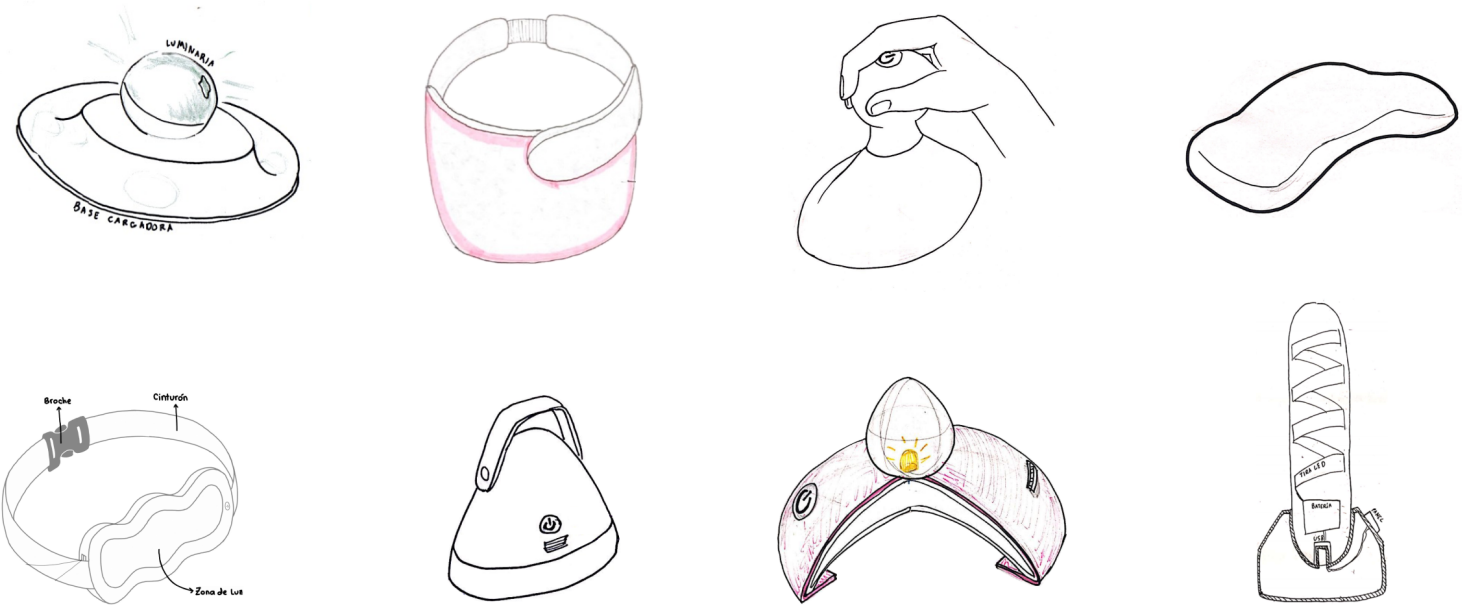

Fig. 2. Bocetos rápidos.

Con esto se elige la propuesta 7, a partir de la cual se continuó trabajando. Se realizaron las variaciones morfológicas que se muestran en la figura 3. Con estos renders de alta fidelidad, se buscó la opinión de 23 posibles personas usuarias a través de una encuesta para conocer su percepción con respecto a las formas. Los resultados de este instrumento permitieron concretar la morfología externa del producto.

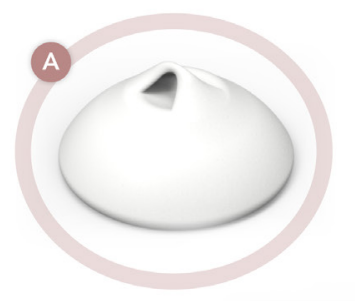

(D)

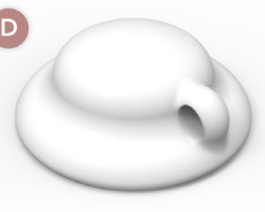

B
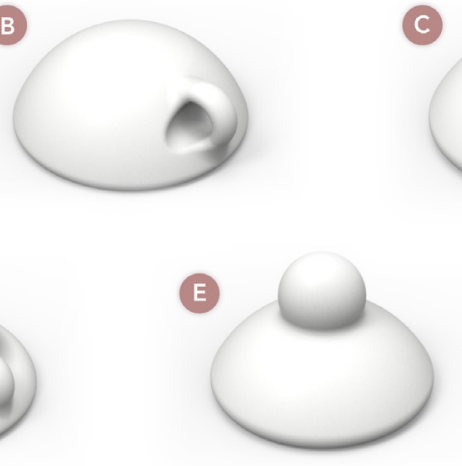

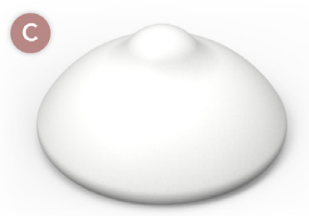

Fig. 3. Propuestas con variaciones morfológicas.

A partir de aquí, se procedió con el detallado técnico. Venus se diseñó para estar compuesta de dos carcasas, unidas por tornillos, elaboradas a partir del proceso de inyección de policarbonato con efecto sand blast. Estas poseen nervaduras que ayudan a mantener todos los componentes internos fijos y proporcionar mayor estructura a la luminaria. El material de la carcasa es resistente a golpes, tracción y torsión con el fin de prolongar su vida útil. 
La configuración interna de la luminaria, como se observa en la figura 4, se basa en un módulo de carga donde se encuentra la conexión con la entrada micro-USB, un botón pulsador de dos estados, una tira LED, colocada alrededor de la estructura, y dos baterías de litio-polímero recargables, cuya energía es suficiente para mantener encendida la luminaria durante 7 horas. Cuando la energía de estas baterías se agota, solamente se debe conectar la luminaria al cargador portátil y esperar 40 minutos hasta que la carga sea completada.
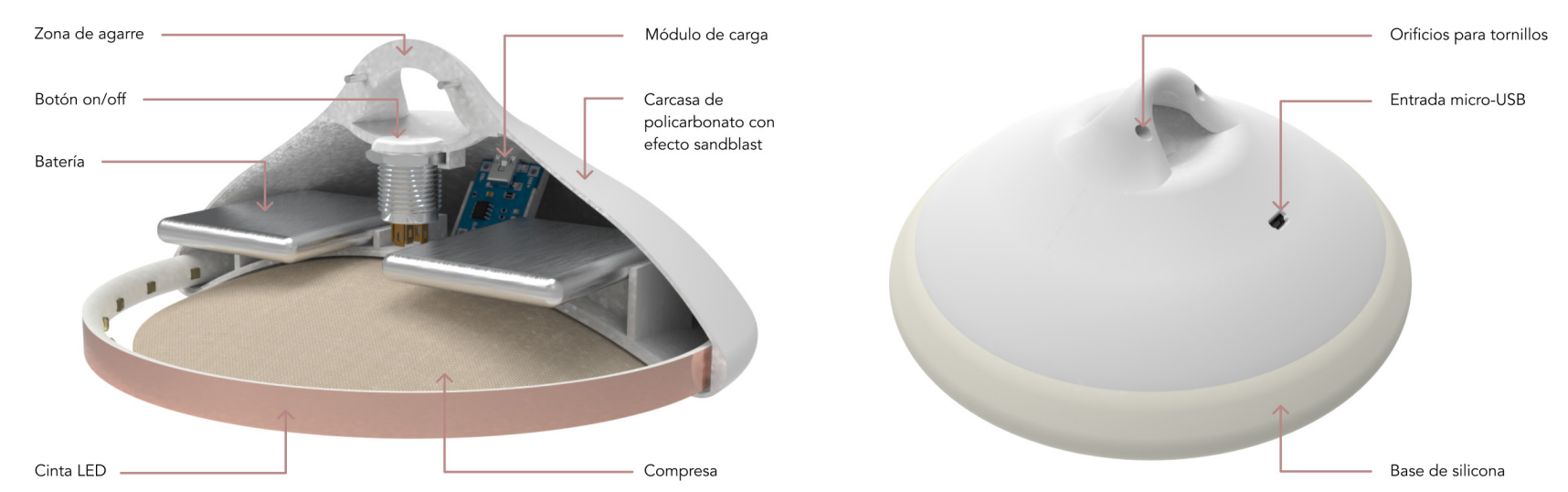

Fig. 4. Configuración de Venus.

Además, en la parte inferior cuenta con una base circular de silicón con alta fricción, realizada a través del proceso de compresión, la cual evita el deslizamiento cuando la luminaria se utiliza, ya sea sobre una superficie plana o sobre el abdomen de la persona usuaria en una posición horizontal. Gracias a la flexibilidad de su material, esta se estira y se coloca alrededor de la parte inferior de las carcasas, de modo que queda un espacio disponible donde se inserta la compresa de manta que se utilizará en la terapia con calor para el alivio del dolor.

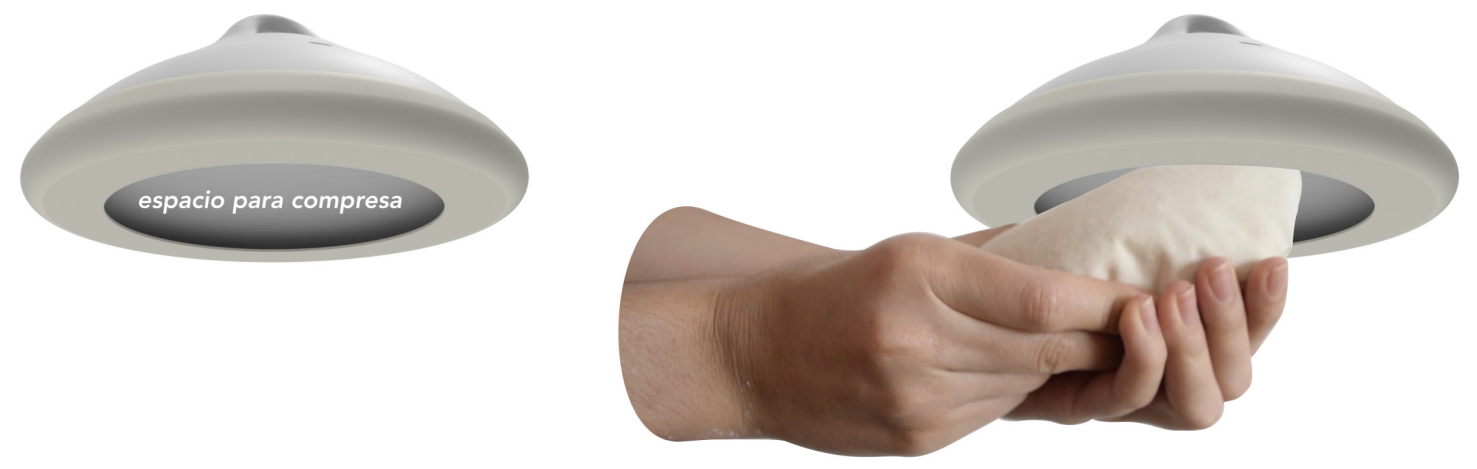

Fig. 5. Compartimento para compresa. 
El cargador es un power bank solar, es decir, una batería externa que trae incorporado un panel solar, el cual transforma la radiación del sol en electricidad, a esto se le conoce como energía fotovoltaica [14]. La carga completa del power bank tiene una duración de 5 horas. Una vez completado el tiempo, la energía se encontrará almacenada dentro del power bank y permitirá que la luminaria pueda ser cargada tanto de día como de noche. Para cargar la luminaria, solamente se debe flexionar la lengüeta del cargador y hacer la conexión entre los puertos hembra y macho, como se observa en la figura 6.
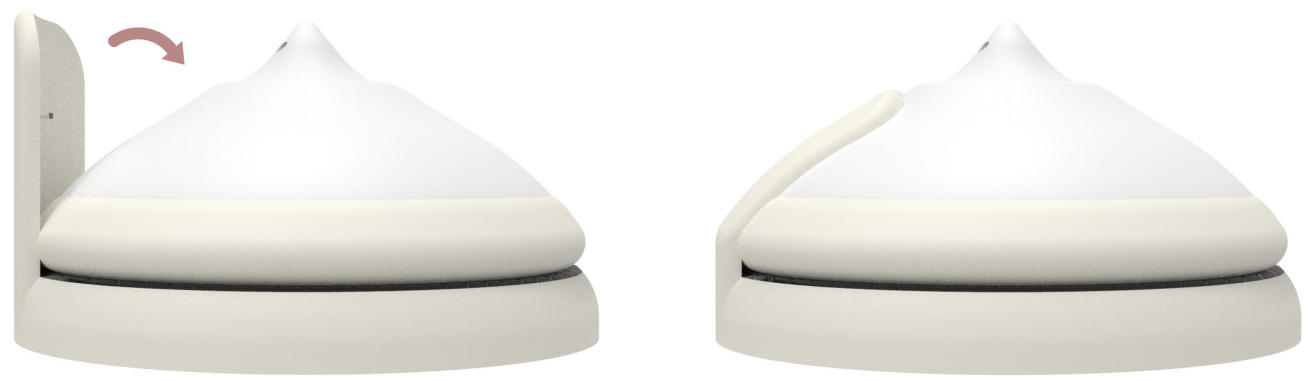

Fig. 6. Flexión de lengüeta.

En la figura 7, se ilustra el proceso de ensamblaje del producto, el cual se diseñó con el fin de posibilitar su desensamblaje y refabricabilidad, al utilizar tornillos para unir la carcasa y facilitar su desensamblaje al finalizar su vida útil y, a su vez, reutilizar sus componentes electrónicos, estos se encuentran accesibles y son fáciles de remover.

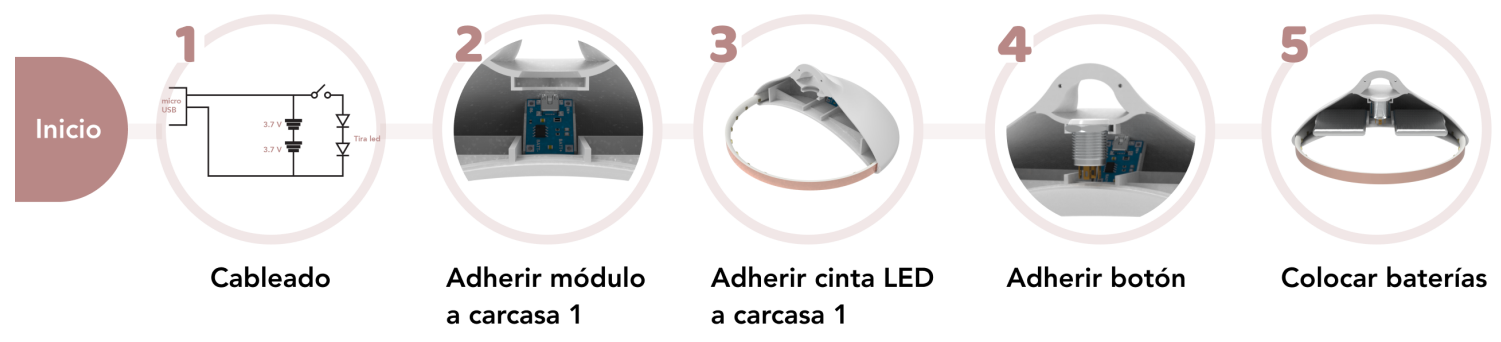

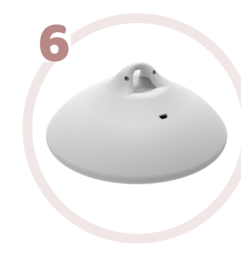

Adherir cinta LED a carcasa 2

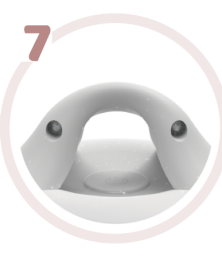

Atornillar

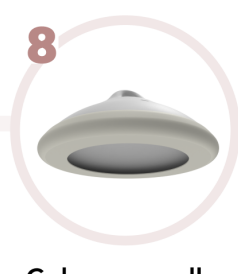

Colocar argolla

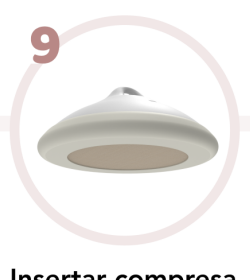

Insertar compresa

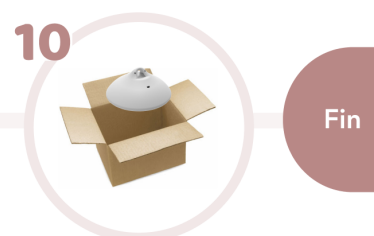

Empaque

Fig. 7. Proceso de ensamblaje. 
En la figura 8, se observa un fotomontaje de Venus, en un contexto de uso doméstico, mientras la persona usuaria se encuentra en posición de descanso. Se puede observar cómo se podría interactuar con la superficie continua de la luminaria y la proyección de su luz tenue.

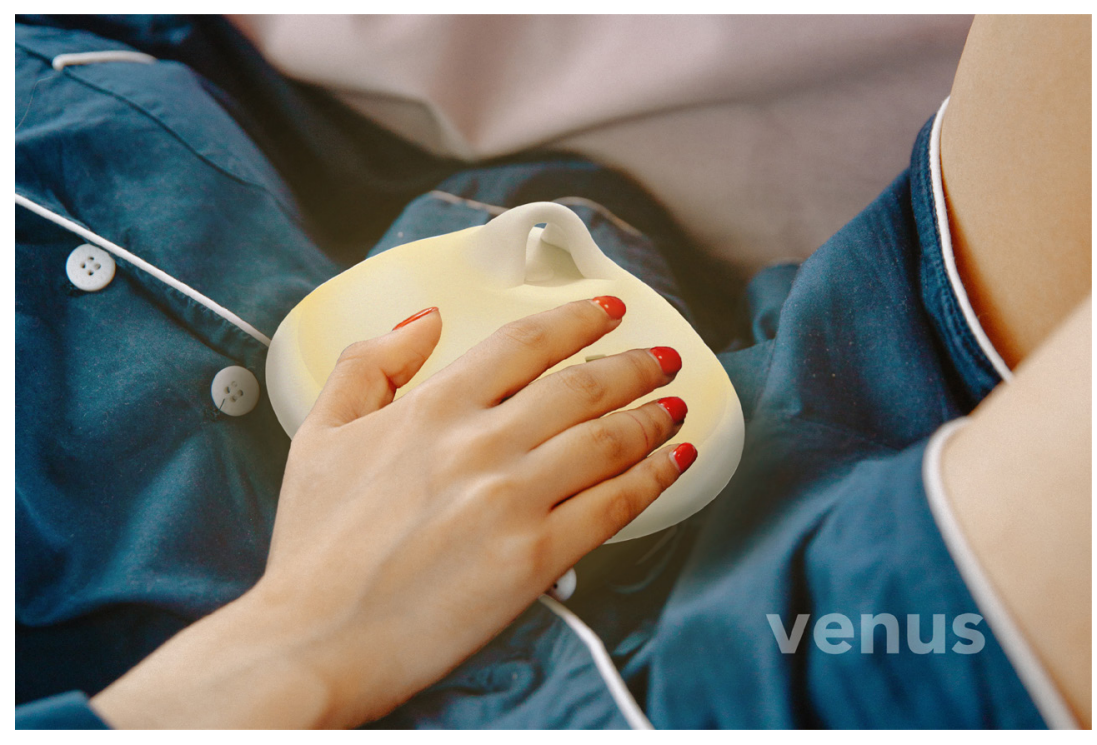

Fig. 8. Fotomontaje realizado en la etapa de simulación con render propio y foto de Polina Zimmerman en Pexels. [14]

\section{Discusión}

Al finalizar la aplicación de la metodología, de la cual se habló anteriormente, se obtuvo un producto que satisface las necesidades planteadas por las diseñadoras al inicio del proyecto. Al analizar la conceptualización final y considerando el factor iterativo presente en el diseño de cualquier producto, se hallaron múltiples oportunidades de mejora para investigar en un futuro, con el fin de pasar del concepto al desarrollo.

Para su mayor comprensión, se procede a dividir en dos categorías dichas oportunidades: aspectos técnicos y experiencia de uso.

\section{Aspectos técnicos}

Posterior a la conceptualización del producto, se identificaron distintos campos que se podrían explorar para mejorar el diseño, entre ellos se discute el cambio de la distribución interna de los componentes electrónicos, con el fin de facilitar el ensamblaje, debido a que la posición inclinada del módulo de carga es de difícil acceso para el obrero de línea encargado del ensamblaje de la luminaria.

Asimismo, se deben realizar pruebas de transmisión de calor, ya que, si el material que compone la carcasa del producto no brinda un buen aislamiento térmico, se debe incorporar una delgada capa aislante en el lugar en el que se posiciona la batería, para que esta no sufra deterioro por 
el calor de la compresa. Además, se debe corroborar que la luminaria transmita únicamente el calor en la dirección deseada.

Finalmente, es necesario realizar pruebas de esfuerzo en los materiales, para considerar si es necesario replantear la disposición de los tornillos en el producto, ya que, en este momento, ambos tornillos se encuentran en un punto de quiebre del producto, debido a que el esfuerzo a la hora de introducir la compresa se concentra en la zona de sujeción. Dicho punto de quiebre se ilustra, a continuación, en la figura 9.

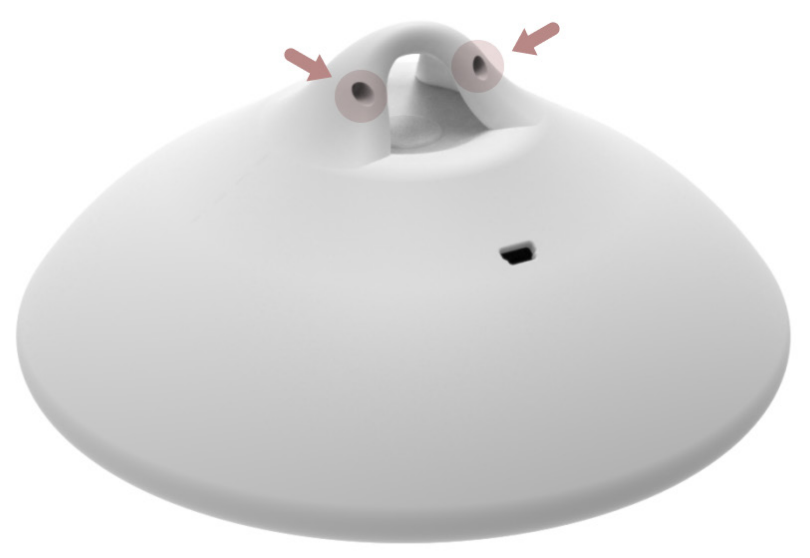

Fig. 9. Posibles puntos de quiebre.

\section{Experiencia de uso}

En el ámbito de uso del producto, se debe realizar un prototipo físico que demuestre que el material en conjunto con la posición de la tira LED brindan el efecto de la luz deseado a la hora de la conceptualización. Una posibilidad de exploración que queda abierta para su futura investigación es la de la meditación en la luz, una técnica utilizada por múltiples culturas a través del tiempo y que podría traer grandes beneficios para las personas usuarias del producto.

\section{Conclusiones}

La conceptualización de Venus permitió identificar necesidades y sistematizar esa indagación, de tal manera que se puntualizan objetivos específicos que guiaron el diseño del producto. Entre los aprendizajes principales de este proyecto, resalta la necesidad de que exista un producto como Venus. Actualmente, no hay un producto en el mercado que pueda satisfacer la necesidad identificada de forma integral como lo hace Venus, a pesar de que existe una incidencia alta de dismenorrea, como se obtuvo de la investigación inicial. Venus tiene características específicas que pueden satisfacer las necesidades de este público meta, lo que lo hace un producto viable con alto potencial de tener éxito en el mercado. Sin embargo, se recomienda desarrollar un 
prototipo experimental que permita obtener datos tanto de la experiencia estética como de los aspectos técnicos, para así corroborar que el producto funcione correctamente y cumpla con su objetivo.

Asimismo, se puede replantear el método de ensamblaje del producto y considerar una manera óptima de llevarlo a cabo sin que exista el riesgo de que se dañe algún componente. Por ejemplo, la colocación de un tornillo en la parte inferior de la luminaria, para evitar que el esfuerzo de colocar la compresa dañe la parte superior en la que se unen las carcasas.

Por último, se recomienda que, para posteriores desarrollos de este producto, se considere el uso de una aplicación móvil donde se pueda llevar un registro completo de la condición de las personas usuarias que utilicen la luminaria y que, de esta manera, el producto se integre como parte esencial del bienestar menstrual.

\section{Referencias bibliográficas}

[1] E. Fairytale, "Mujer meditando en postura de loto," 2020, Pexels. [En línea]. Disponible: https://www.pexels.com/es-es/foto/mujer-meditando-en-postura-de-loto-3822583/

[2] L. Zanin, C. Correa, A. Paez, y M. De Bortoli, "Ciclo menstrual: sintomatología física y psicológica. Un estudio preliminar", presentado en II Congreso Internacional de Investigación y Práctica Profesional en Psicología, las XVII Jornadas de Investigación y el Sexto Encuentro de Investigadores en Psicología del MERCOSUR. Buenos Aires, Argentina, Nov. 22-24, 2010. Disponible: https://www.aacademica.org/000-031/305.pdf

[3] G. Grandi, S. Ferarri, A. Xholli, M. Canoletta, F. Palma, C. Romani, A. Volpe, y A. Cagnacci, "Prevalence of menstrual pain in young women: What is dysmenorrhea?" Journal of Pain Research vol.5, pp. 169, Jun, 2012. Acceso: Dic, 20, 2020, doi: 10.2147/JPR.S30602. [En línea]. Disponible: https://www.ncbi.nlm.nih.gov/pmc/articles/PMC3392715/.

[4] M. Ortiz y L. Romero, “Dismenorrea: dolor crónico cíclico más común y mal tratado en las mujeres", Medwave, vol. 13, no. 03, 2013. doi: 10.5867/medwave.2013.03.5656 [En línea] Disponible: https://www.medwave.cl/link.cgi/Medwave/PuestaDia/Practica/5656.

[5 Reyes, "Tratamiento fisioterapéutico en la dismenorrea primaria", Tesis M.S, Depto. ciencias médicas, Univ. UCA, Cadiz, Andalucía, España, 2018. [En línea]. Disponible: https://www.fisiofocus.com/es/beca-tfg/img/tfg/Sara\%20Reyes\%20Fornell_ Tratamiento\%20fisioterapeutico\%20en\%20la\%20dismenorrea\%20primaria.pdf

[6] Z. Rakhshaee, "Effect of Three Yoga Poses (Cobra, Cat and Fish Poses) in Women with Primary Dysmenorrhea: A Randomized Clinical Trial", Journal of Pediatric and Adolescent Gynecology, vol. 24, no. 4, pp. 192-196, 2011. [En línea] Disponible: 10.1016/j. jpag.2011.01.059.

[7] M. Proctor and C. Farquhar, "Diagnosis and management of dysmenorrhoea", BMJ, vol. 332, no. 7550, 2006. doi: 10.1136/bmj.332.7550.1134 [En línea] Disponible: https:// www.ncbi.nlm.nih.gov/pmc/articles/PMC1459624/. 


\section{revistaIDI+}

[8] P. Hekkert, Design aesthetics: Principles of pleasure in product design., Delft: Psychology, 2006.

[9] L. Santos, "Factores de riesgo ergonómico en técnicos de atención médica prehospitalaria en una organización en México", Tesis M.S, Escuela Nacional de Medicina y homeopatía, Instituto Politécnico Nacional, México, D.F., 2016. [En línea]. Disponible: https://tesis.ipn.

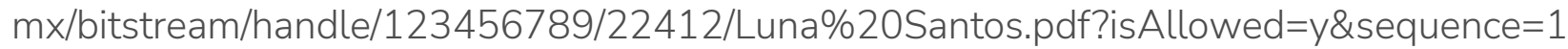

[10] V. Garro, M. Thuel y V. Robles, "Dismenorrea primaria en las adolescentes: manejo en la atención primaria", Revista Médica Sinergia, vol. 4, no. 11, 2019. doi: 10.31434/rms. v4i11.296. [En línea] Disponible: https://revistamedicasinergia.com/index.php/rms/article/ view/296.

[11] J. A. Diego-Mas, "Evaluación de la manipulación manual de cargas mediante GINSHT," 2015. [En línea] Disponible: https://www.ergonautas.upv.es/metodos/ginsht/ginshtayuda.php

[12] J. Panero, M. Zelnic, "La dimensión humana. Tablas antropométricas" en Las dimensiones humanas en los espacios interiores, 7ma ed. Barcelona, España: Ediciones G.Giii, 1996, cap. B, sec. 2, pp. 90. [En línea]. Disponible: https://vdocuments.net/las-dimensioneshumanas-en-los-espacios-interiores-58a3556c5af32.html

[13] J. Barbosa, F. Trujillo, P. Velásquez, y J. Castellanos, "Estudio para el uso de la tecnología solar fotovoltaica”, Revista Ingeniería Solidaría, vol. 6, no. 10-11, 2010. Disponible: https://revistas.ucc.edu.co/index.php/in/article/download/453/458/

[14] P. Zimmerman, “Mujer-conmovedor-enfermo-indispuesto," 2020, Pexels. [En línea]. Disponible: https://www.pexels.com/es-es/foto/mujer-conmovedor-enfermoindispuesto-3958565/ 